\title{
Phenomenological based Model of Hydrogen production using an Alkaline self-pressurized Electrolyzer
}

\author{
M. David ${ }^{1,2}$, H. Alvarez ${ }^{3}$, C. Ocampo-Martinez ${ }^{2}$ and R. Sánchez-Peña ${ }^{1}$
}

\begin{abstract}
The aim of this work is to develop the phenomenological based modeling of a self-pressurized alkaline electrolyser with the objective of predicting the cross-contamination of the gases produced. A proposed model, built in Matlab ${ }^{\circledR}$, represents the dynamical evolution in real electrolysers, and anticipates operational variables: level, pressure and all concentrations. Dynamic responses in the concentrations of the electrolytic cell, and variations in both level and pressure at the chamber due to the change in current and diffusivity, are reported. The equations by which the variables can be computed are also presented. The proposed model is ready for the corresponding adjustment of parameters based on experimental measurements taken from an available prototype and through a suitable identification process.
\end{abstract}

\section{INTRODUCTION}

The world economy is constantly expanding. There are two influencing factors related to that expansion: the population growth and progress in personal comfort. Both factors affect the current fossil economy by increasing consumption and generating greater amounts of greenhouse gases (GHG). This situation is widely accepted as critical, so worldwide environmental impact studies and environmental protection policies are generated.

To achieve high integration of renewable energy, it is necessary to have the ability to accumulate the excess of energy to be consumed at a time when consumption exceeds production. Among the methods of energy storage, hydrogen production currently takes relevance due to its energy density, high capacity and portability [1], [2]. Among all the methods of hydrogen production, electrolysis holds a dominant position on the use of the fluctuating electricity from renewable energy. This fact is given due to its ease of connection with these sources, production of high purity hydrogen and current infrastructure. For these reasons, it is the interest of the Buenos Aires Institute of Technology (ITBA) to investigate electrolysis, particularly the self-pressurized alkaline method, which allows to reduce the compression energy consumption [3]. So far four prototypes have been developed at the university, following now with the modelling and control design to optimize their production capacity.

\footnotetext{
${ }^{1}$ Instituto Tecnológico Buenos Aires (ITBA) and Consejo Nacional de Investigaciones Científicas y Técnicas (CONICET), Ciudad Autnoma de Buenos Aires, Argentina

${ }^{2}$ Automatic Control Department, Universitat Politècnica de Catalunya, Institut de Robòtica i Informàtica Industrial (CSIC-UPC), Barcelona, España

${ }^{3}$ Processes and Energy Department, National University of Colombia, Kalman Research Group, Medellín, Colombia

This work has been partially funded by the Spanish State Research Agency through the Mara de Maeztu Seal of Excellence to IRI (MDM2016-0656) and the Cheerful CSIC project (MHE-200065).
}

While it is true that the principle of operation of alkaline cells has been described widely by several authors, most of them focused on the stationary regime and presented empirical analytical relationships from the adjustment of a specific electrolyzer. In 2003, Ulleberg [4] proposed a model based on thermodynamic concepts and heat transfer to obtain the voltage of the package, the gas flow produced and the thermal equilibrium of the system, all of them as a function of the imposed current. These results were validated at the PHOEBUS plant in Jlich, where photovoltaic cells, hydrogen production and storage tests were carried out [5]. Next, Amores et al. [6] add the influence of the concentration of electrolyte and the distance between electrodes. In the same direction, Ursa and Sanchis [7] start from the same thermodynamic setup to define the ideal water dissociation voltage. They build an electric model of over-voltages, which is one of the few cases of dynamic equations, although only limited to the electrical part. Moreover, the work done by Havre and Tommerberg [8] presents a simplified model of the dynamics of an electrolyzer that serves as a first step. The most complete model describing the dynamic behaviour is presented by Roy in his doctoral thesis [9], who inquires about the installation of electrolyzers with renewable energies. However, this model was constructed mainly from experimental adjustment equations, losing phenomenological details that are in fact recovered in the present work.

Concerning the contamination of the produced gases, empirical adjustments can be found, as is the case of the work done by Hug [10] or the research line carried out by the Max Planck Institute for Iron Research [11], [12], [13]. Those reported works study the diffusion through the membrane that separates half cells. However, a model integrating cell behaviours and transport phenomena taking place in the assembly as a whole has not been found so far in the literature. To fill this gap, the main contribution of this paper relies on the proposal of a phenomenologicalbased semi-physical model (PBSM) that feeds from all this information, and describes the phenomena that occur within the electrolyzer. The availability of this model will allow a more accurate idea of the dynamics and even set guidelines for design improvement in future prototypes. The phenomenological based approach gives in addition the possibility of refinements of the model by the use of better formulations to calculate model parameters.

The remainder of the paper is structured as follow: in Section II] the work methodology is explained along with the resulting model. In Section III] some simulations are presented and analyzed. Finally, Section IV draws the main 
conclusions of the paper.

\section{BUILDING OF A PBSM OF HYDROGEN PRODUCTION BY WATER ELECTROLYSIS}

The PBSM takes its structure from conservation principles, allowing the use empirical equations to formulate model parameters. In this way, the best of white-box and black box models is considered to obtain a gray-box model [14], [15]. The proposed methodology, referred to the work in [16], is detailed in the following.

\section{II-A. Process description and model objective}

A scheme of the equipment designed and installed at the Electrolyzer of the Hydrogen Laboratory (ELH) of ITBA is illustrated in Figure 1.(a). This equipment has a pressurized tank containing a package of 15 alkaline electrolytic cells as illustrated in Figure 1.(b), two gas separation chambers, two refrigeration systems, two $K O H$ solution circuits, and one water make-up pump. The symmetry of the assembly is respected in the system modelling allowing a parallel implementation of the equations.

The gas outlet lines are driven by two motorized valves that allow to control both the pressure of gases and levels in both chambers of the ELH. As shown in Figure 1](c), water is produced at the anode while consumed at the cathode, then there is a change in the concentration of $K O H$. To avoid this phenomenon, both circuits are communicated through the pressure tank, resulting in the equalization of internal and external pressures as well as the equalization of $\mathrm{KOH}$ concentration due to the water make-up.

\section{II-B. Modeling hypothesis}

The model objective is to predict the contamination of each gas stream with the other gas due to the membrane permeability according to changes in both pressure and current. In addition, the pressure in both sides of the electrolyzer should be also predicted. The operation can be split in two major phenomena: the gas production at each semi-cell and the gas separation and compression in the gas separation chamber. Other phenomena, such as heat and momentum exchange are simple, although they require a mathematical formulation for their evaluation. These phenomena are taken into account in the model, although they are not presented in this paper. For more details about their mathematical formulation, the reader can refer to cited literature.

The major hypotheses formulated for the model development are reported next. The water in contact with the electrodes participates in two chemical reactions in the electrolytic cell (Figure 1.(c)), one at each electrode, driven by the electric current symbolized by the flow of electrons $e^{-}$:

$$
\begin{aligned}
& 2 \mathrm{H}_{2} \mathrm{O}_{(l i q)}+2 e^{-} \rightarrow \mathrm{H}_{2}(g)+2 \mathrm{OH}_{(a q)}^{-}, \\
& 2 \mathrm{OH}_{(a q)}^{-} \rightarrow \frac{1}{2} \mathrm{O}_{2(g)}+2 e^{-}+\mathrm{H}_{2} \mathrm{O}_{(l i q)} .
\end{aligned}
$$

Each reaction occurs in a half cell, then there is no direct mixing of gases in that space. However, the membrane that separates the half cells is slightly permeable to dissolved gases, therefore a first focus of cross-contamination of gases appears. All excess of gas that produces the reaction over the limit of solubility is transported in the liquid as small bubbles. The separation of the gas bubbles is achieved in the separation chamber. The solution saturated in the gas at the chamber pressure, but degassed, is removed from the chamber through the recirculation pump towards the cell.

The assumptions completing the modeling hypothesis previously stated are: $i$ ) perfect agitation in all process systems (PSs) except gassed liquid in the separation chamber, ii) the half cells always operate at full volume without gas accumulation, iii) all the ion $\mathrm{OH}^{-}$is produced or consumed within the half cells, i.e., there is no $\mathrm{OH}^{-}$in any other stream, $i v)$ spatially uniform temperature throughout the device, $v$ ) temporarily constant temperature due to the action of the cooling system, vi) the recirculation pumps allow to overcome the friction in the system and guarantee the flow between the half cells and the separation chambers, vii) the gas mixture in the upper part of the separation chambers is considered as an ideal gas, and viii) gas bubbles produced in the half cells are contaminated with dissolved impure gas only on the free surface of the liquid at the separation chamber.

\section{II-C. Process system definition}

Figure 2 shows the process systems (PSs) that are taken into account to build the model. The number of each PS is placed in Roman next to each box. All the 16 PSs resulting from the balances are reported. To simplify the modeling, those PSs showing a quite simple action are formulated with algebraic expressions. The process symmetry is also exploited for model complexity reduction. The cathodic half where $\mathrm{H}_{2}$ is produced is presented in the next subsection. For the following PSs, all balances must be raised, i.e., PS I, III, VII and, finally, $\mathrm{PS}_{X I I I}$, which is not symmetric. As already mentioned, the other PSs have trivial models, then no balance is performed there. For the $\mathrm{PS}_{I}$, the only one with a chemical reaction, all balances are worked on a molar basis instead of using the mass base.

\section{II-D. Application of the conservation principle}

For a better understanding, two balance equations (3) and (5) are developed in detail, while the complete model structure reported in Table $\mathrm{I}$ is detailed in [17]. The conservation law will be applied to the PSs of interest mentioned in the previous step. Here, the total mass balance and one of the balances per component for $\mathrm{PS}_{I}$ are presented, as an explanation of the procedure. Next, the complete set of final equations is summarized in Section II-E In this way, the basic structure of the model is obtained, which will allow the model objectives fulfilment (Section II-A).

II-D.1. Total Mass Balance (TMB): The global balance, illustrated in Figure 2, is stated as follows:

$\frac{d N_{I}}{d t}=\dot{n}_{1}+\dot{n}_{6}-\dot{n}_{21}-\dot{n}_{3}-\dot{n}_{5}+\dot{n}_{22}+r_{1} \sum_{i} \sigma_{i, 1}$,

with $N_{I}$ the total number of moles in the space containing the solution in the anodic half cell $\dot{n}_{i}$ for the $i$-th current, and 


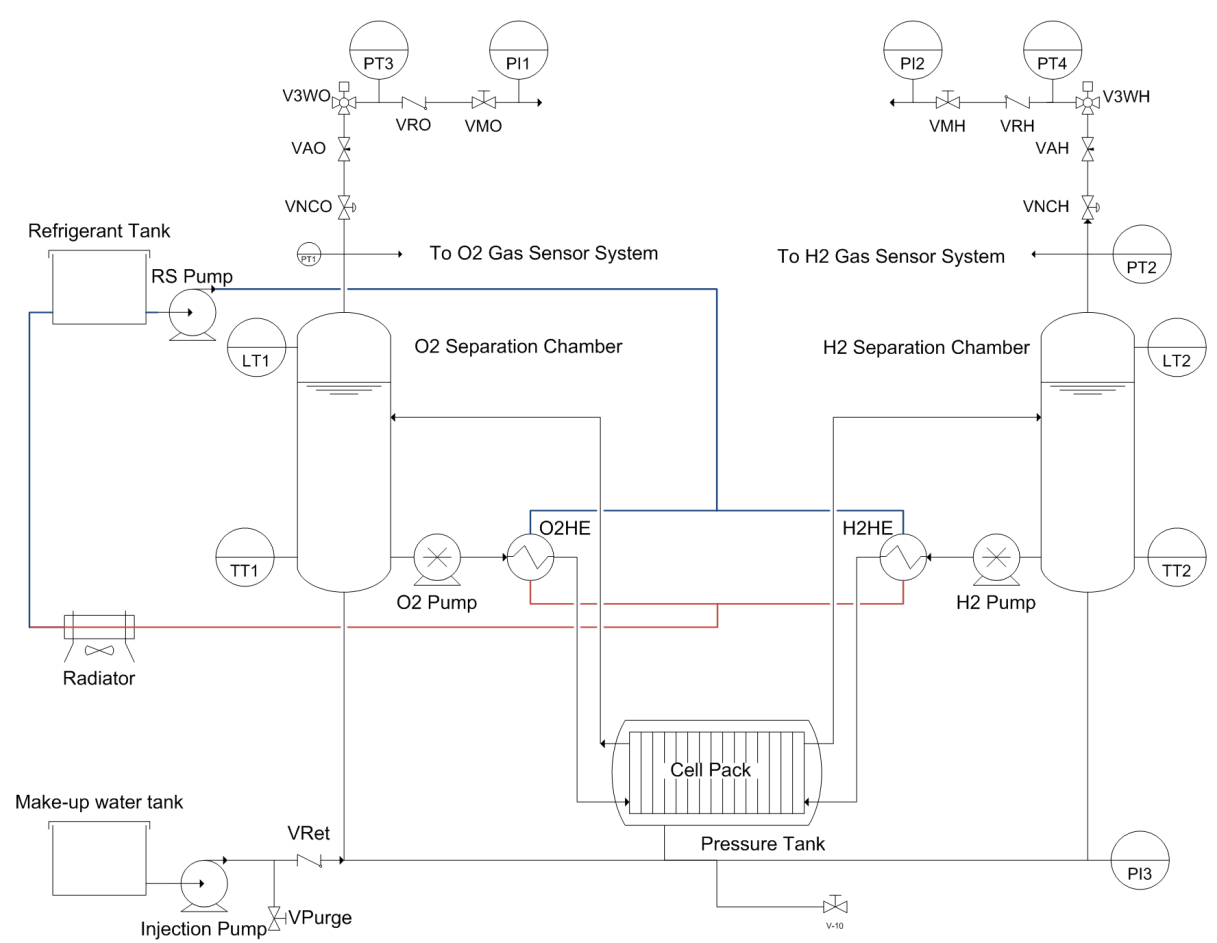

(a)

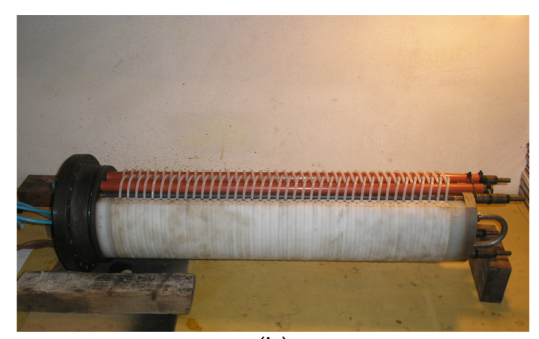

(b)

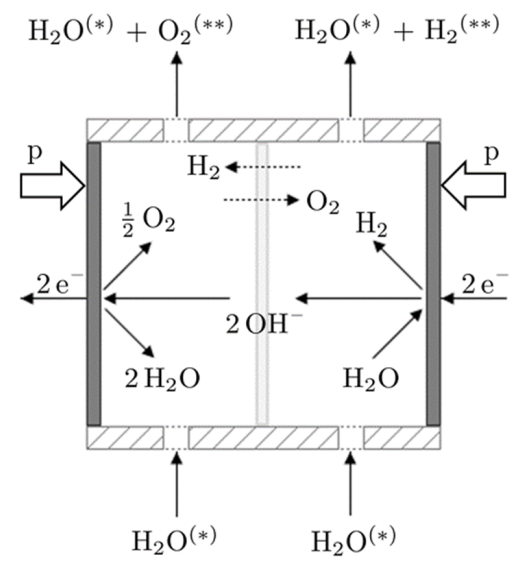

(c)

Fig. 1. (a) P\&ID diagram of the Electrolyzer of the Hydrogen Laboratory (ELH), (b) photo of the real cell package, and (c) scheme of the electrolytic cell with reactions. $\mathrm{H}_{2} \mathrm{O}^{(*)}$ represents $\mathrm{KOH}$ solution and $\mathrm{O}_{2}^{(*)}$ and $\mathrm{H}_{2}^{(* *)}$ represent outputs that are contaminated with $\mathrm{H}_{2}$ and $\mathrm{O}_{2}$, respectively.

$r_{1}$ is the speed at which the half-cell electrochemical reaction (1a) proceeds. The final sum is about the stoichiometric coefficients $\sigma_{i, 1}$ of the species $i$ in the same reaction.

The total number of moles can be expressed as $N_{I}=\bar{\rho} V_{m i x, I}$, where $\bar{\rho}$ is the molar density of the mixture in $\frac{\mathrm{kg}}{\mathrm{kmol}}$ and $V_{m i x, I}$ is the volume of the entire mixture (liquid and gas bubbles) contained in the $\mathrm{PS}_{I}$. With the assumption of constant volume of the half cell, applying the derivative to replace it in equation (2) and considering that the molar flow of electrons is equal to the molar flow of $\mathrm{OH}^{-}$, the final balance equation is as follows:

$$
\frac{d \bar{\rho}_{3}}{d t}=\frac{1}{V_{m i x, I}}\left[\dot{n}_{1}+\dot{n}_{6}-\dot{n}_{3}-\dot{n}_{5}+r_{1} \sum_{i} \sigma_{i, 1}\right] \text {. }
$$

II-D.2. Component Mass Balance for hydrogen: The balance for $H_{2}$ in $\mathrm{PS}_{I}$ is

$$
\begin{aligned}
\frac{d N_{H_{2}, I}}{d t}= & x_{H_{2}, 1} \dot{n}_{1}+x_{H_{2}, 6} \dot{n}_{6}-x_{H_{2}, 21} \dot{n}_{21} \\
& -x_{H_{2}, 3} \dot{n}_{3}-x_{H_{2}, 5} \dot{n}_{5}+r_{1} \sigma_{H_{2}, 1},
\end{aligned}
$$

where $N_{H_{2}, I}$ is the mass of hydrogen (in moles) contained in the $\mathrm{PS}_{I}, x_{H_{2}, i}$ is the molar fraction of $\mathrm{H}_{2}$ in $\frac{\mathrm{kmol} \mathrm{H}}{\mathrm{kmolmix}}$ with respect to the $i$-th current and $\sigma_{H_{2}, 1}$ is the stoichiometric coefficient of hydrogen in the balanced electrochemical reaction (1a). It should be clarified that $x_{H_{2}, i}$ for current 3 and eventually for current 5 , if the separation chamber is not operating correctly, refers to both dissolved and bubble hydrogen. Moreover, taking into account that the $\mathrm{H}_{2}$ concentrations in currents 6 and 21 are zero, i.e., $x_{H_{2}, 6}=$ $x_{H_{2}, 21}=0$, that the stoichiometric coefficient $\sigma_{H_{2}, 1}=1$ and that the outgoing flux that passes through the membrane $\dot{n}_{5}$ is composed only of $H_{2}$. Therefore,

$\frac{d x_{H_{2}, 1}}{d t}=\frac{1}{N_{I}}\left[x_{H_{2}, 1} \dot{n}_{1}-x_{H_{2}, 3} \dot{n}_{3}-\dot{n}_{5}+r_{1}-x_{H_{2}, I} \dot{N}_{I}\right]$,

where, by perfect agitation hypothesis, the concentration of output flow 11 can be considered equal to the compositions into this $\mathrm{PS}_{I}$.

\section{II-E. Structure, parameters and constants}

The balance equations of the basic structure of the model are presented in Table I, and the list of symbols is reported in Table II. For further details, please refer to [17].

\section{II-F. Degrees of freedom analysis}

A solvable model is obtained when its degrees of freedom (the difference between the number of unknown terms and equations) is null. In this case, the amount of unknown terms is the sum of 21 model variables and 38 model parameters, a total of 59 unknown terms. On the other hand, there are 21 equations from the model basic structure and 38 for model parameter evaluation, yielding 59 equations. Hence, the degree of freedom of the model is null, allowing to compute a model solution. 


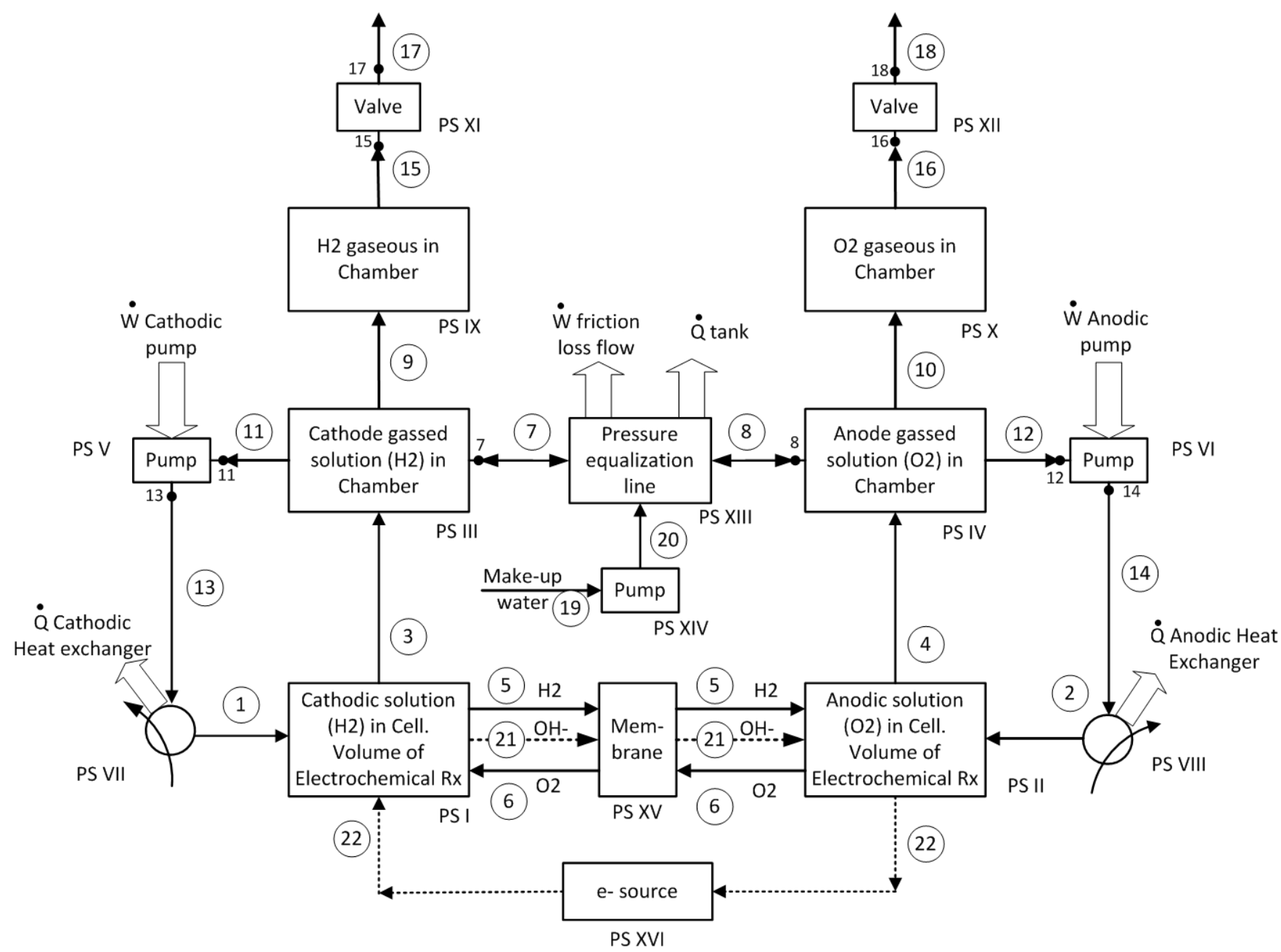

Fig. 2. Flow diagram with the PSs numbered in Roman. Mass flows are identified with numbers within circles

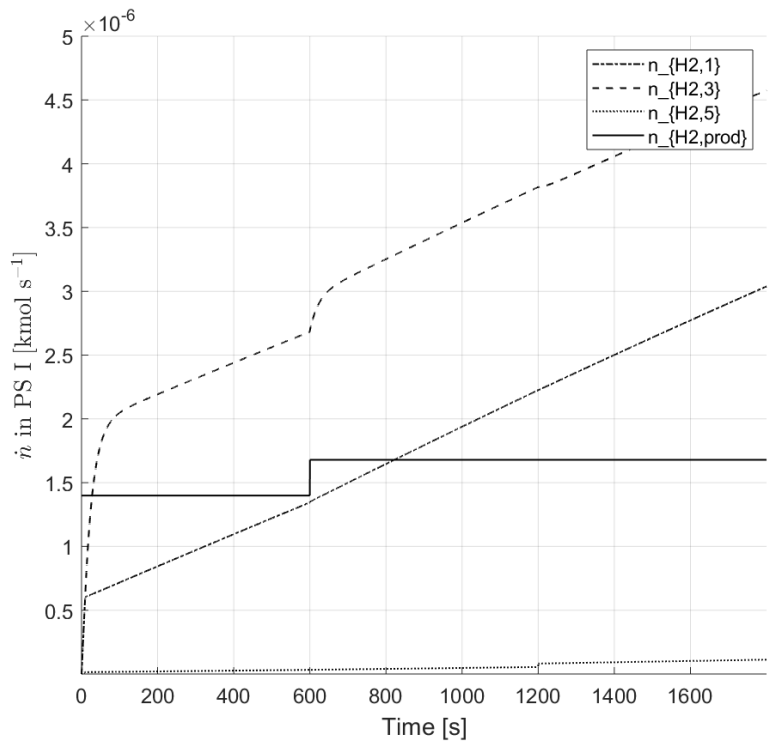

(a)

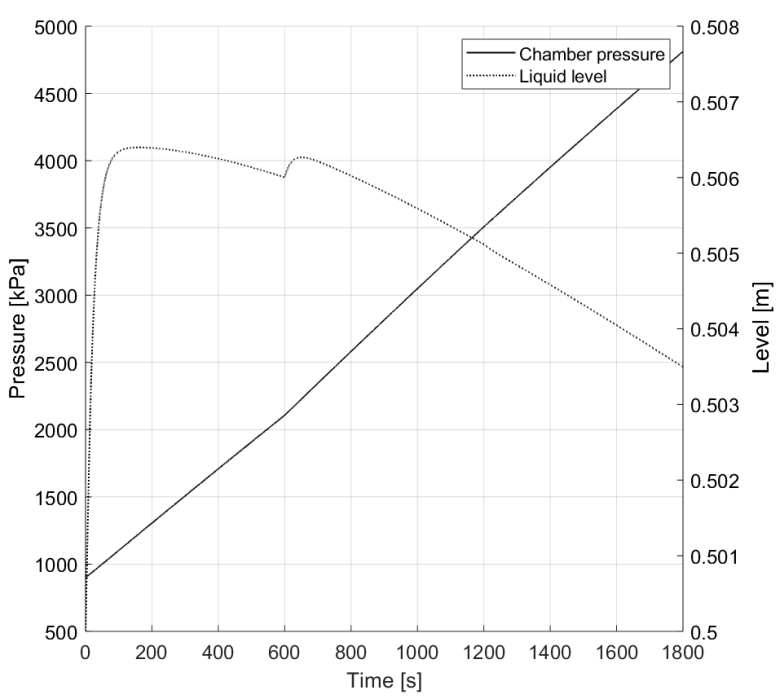

(b)

Fig. 3. (a) Dynamic response of molar flows in the electrolytic cell. (b) Pressure and gassed liquid level response in the gas separation chamber. 
TABLE I

BALANCES EQUATIONS FORMING THE MODEL BASIC STRUCTURE.

\begin{tabular}{|c|c|c|}
\hline \# & Equation & $\begin{array}{l}\text { Process } \\
\text { System }\end{array}$ \\
\hline 1 & $\frac{d \bar{\rho}_{3}}{d t}=\frac{1}{V_{m i x, I}}\left[\dot{n}_{1}+\dot{n}_{6}-\dot{n}_{3}-\dot{n}_{5}+r_{1} \sum_{i} \sigma_{i, 1}\right]$ & $P S_{I}$ \\
\hline 2 & $\frac{d x_{H_{2}, 3}}{d t}=\frac{1}{N_{I}}\left[x_{H_{2}, 1} \dot{n}_{1}-x_{H_{2}, 3} \dot{n}_{3}-\dot{n}_{5}+r_{1}-x_{H_{2}, 3} \dot{N}_{I}\right]$ & $P S_{I}$ \\
\hline 3 & $\frac{d x_{O_{2}, 3}}{d t}=\frac{1}{N_{I}}\left[x_{O_{2}, 1} \dot{n}_{1}+\dot{n}_{6}-x_{O_{2}, 3} \dot{n}_{3}-x_{O_{2}, 3} \dot{N}_{I}\right]$ & $P S_{I}$ \\
\hline 4 & $\dot{n}_{21}=-2 r_{1}$ & $P S_{I}$ \\
\hline $\begin{array}{l}5 \\
6\end{array}$ & $\begin{array}{l}\dot{n}_{22}=\dot{n}_{22} \\
\frac{d M_{I I I}}{d t}=\dot{m}_{3}+\dot{m}_{7}-\dot{m}_{9}-\dot{m}_{11}\end{array}$ & $P S_{I}$ \\
\hline 7 & $\frac{d w_{H_{2}, I I I}}{d t}=\frac{1}{M_{I I I}}\left[w_{H_{2}, 3} \dot{m}_{3}+w_{H_{2}, 7} \dot{m}_{7}-w_{H_{2}, 9} \dot{m}_{9}-w_{H_{2}, 11} \dot{m}_{11}-w_{H_{2}, 11} \dot{M}_{I I I}\right]$ & $P S_{I I I}$ \\
\hline 8 & $\frac{d w_{O_{2}, I I I}}{d t}=\frac{1}{M_{I I I}}\left[w_{O_{2}, 3} \dot{m}_{3}+w_{O_{2}, 7} \dot{m}_{7}-w_{O_{2}, 9} \dot{m}_{9}-w_{O_{2}, 11} \dot{m}_{11}-w_{O_{2}, 11} \dot{M}_{I I I}\right]$ & $P S_{I I I}$ \\
\hline 9 & $\dot{m}_{11}=\dot{m}_{13}$ & $P S_{V}$ \\
\hline 10 & $0=\eta_{1} \hat{W}_{1}-\frac{P_{13}-P_{11}}{\rho_{L, 11}} \Rightarrow \dot{m}_{13}=f\left(\eta_{1} \hat{W}_{1}-\frac{P_{13}-P_{11}}{\rho_{L, 11}}\right)$ & $P S_{V}$ \\
\hline $\begin{array}{l}11 \\
12 \\
13 \\
14\end{array}$ & $\begin{array}{l}w_{H_{2}, 13}=w_{H_{2}, 11} \\
w_{O_{2}, 13}=w_{O_{2}, 11} \\
w_{H_{2}, 1}=w_{H_{2}, 13} \\
w_{O_{2}, 1}=w_{O_{2}, 13}\end{array}$ & $\begin{array}{l}P S_{V} \\
P S_{V} \\
P S_{V I I} \\
P S_{V I I}\end{array}$ \\
\hline 15 & $\frac{d P_{15}}{d t}=\frac{R T}{\mathfrak{M}_{g} A_{T} L_{g, I X}}\left(\dot{m}_{9}-\dot{m}_{15}\right)-\frac{P}{L_{g, I X}} \dot{L}_{g, I X}$ & $P S_{I X}$ \\
\hline 16 & $\frac{d w_{H_{2}, 15}}{d t}=\frac{1}{M_{I X}}\left[w_{H_{2}, 9} \dot{m}_{9}-w_{H_{2}, 15} \dot{m}_{15}-w_{H_{2}, 15} \dot{M}_{I X}\right]$ & $P S_{I X}$ \\
\hline 17 & $\frac{d w_{O_{2}, 15}}{d t}=\frac{1}{M_{I X}}\left[w_{O_{2}, 9} \dot{m}_{9}-w_{O_{2}, 15} \dot{m}_{15}-w_{O_{2}, 15} \dot{M}_{I X}\right]$ & $P S_{I X}$ \\
\hline 18 & $\dot{m}_{17}=\dot{m}_{15}$ & $P S_{X I}$ \\
\hline $\begin{array}{l}19 \\
20\end{array}$ & $\begin{array}{l}\dot{V}_{17}=u_{1} C_{v, 1} \sqrt{\frac{P_{17}-P_{15}}{\rho_{g}, X I}} \\
\dot{m}_{7}=\dot{m}_{8}\end{array}$ & $\begin{array}{l}P S_{X I} \\
P S_{X I I I}\end{array}$ \\
\hline 21 & $0=\frac{P_{8}-P_{7}}{\rho_{L}}-h_{f, 8 \rightarrow 7} \Rightarrow \dot{m}_{8}=f\left(h_{f, 8 \rightarrow 7}-\frac{P_{8}-P_{7}}{\rho_{L}}\right)$ & $P S_{X I I I}$ \\
\hline
\end{tabular}

TABLE II

LIST OF SYMBOLS

\begin{tabular}{llll}
\hline \hline \multicolumn{1}{c}{ Symbol } & Name & Symbol & Name \\
\hline $\bar{\rho}_{i}$ & Molar density of stream i & $V_{m i x, I}$ & Volume in $P S_{I}$ \\
$\dot{n}_{i}$ & Molar flow in stream i & $r_{j}$ & Reaction speed of reaction j \\
$\sigma_{X, j}$ & Stoichiometric coefficient of X in reaction $\mathrm{j}$ & $x_{X, 3}$ & Concentration of species X in molar fraction \\
$N_{I}$ & Total moles in $P S_{I}$ & $M_{I I I}$ & Total mass in $P S_{I I I}$ \\
$\dot{m}_{i}$ & Mass flow in stream i & $w_{X, I I I}$ & Concentration of species X in mass fraction \\
$\eta_{1}$ & Pump efficiency & $\hat{W}_{1}$ & Specific work of the Pump \\
$P_{j}$ & Pressure in point $\mathrm{j}$ & $\rho_{L, i}$ & Mass density in stream i \\
$R$ & Ideal gas constant & $T$ & System temperature \\
$\mathfrak{M}_{X}$ & Molar mass of species X & $A_{T}$ & Chamber cross area \\
$L_{g, I X}$ & Height of gas volume & $\rho_{g, X I}$ & Mass density of gas in $P S_{X I}$ \\
$\dot{V}_{i}$ & Volumetric flow in stream i & $h_{f, a \rightarrow b}$ & Friction energy loss from $a$ to $b$ \\
\hline
\end{tabular}

\section{MODEL SOLUTION AND RESULT ANALYSIS}

The model extended structure is solved using Matlab ${ }^{\circledR}$. Using the code obtained in this work, which is simple due to the lumped parameter characteristics of PBSM, several operative conditions of the electrolyzer were simulated. Those conditions allow to validate the behavior of this PBSM regarding operation data taken from the actual assembly. Two step disturbances where applied at $600 \mathrm{~s}$ and $1200 \mathrm{~s}$ of simulation. The order of such disturbances is: first an electric current step from $20 \mathrm{~A}$ to $24 \mathrm{~A}$, and next an increase of $50 \%$ in membrane diffusivity. Figure 3 a presents the open-loop dynamic behavior of molar flow in the electrolytic cell. Four observable facts can be enumerated in the graph: $(i)$ there is a step in the production of hydrogen corresponding to the increase in the current, (ii) the concentration of $\mathrm{H}_{2}$ at the entrance of the cell corresponds to the increase in the solubility of the gas compared to the growth of the pressure in the system, (iii) the molar current of contamination towards the anodic half cell slowly grows due also to the greater quantity of dissolved gas, and (iv) $\mathrm{H}_{2}$ at the exit of the half cell is, except during transients, the difference between the other three flows.

In Figure $3 \mathrm{~b}$, the changes of pressure in $\mathrm{H}_{2}$ separation chamber are illustrated with the gas exit valve closed. The pressure increases as gas accumulates in the upper part of the chamber. It can be seen that when there is an increase in electric current, the rate of change of the pressure slightly 


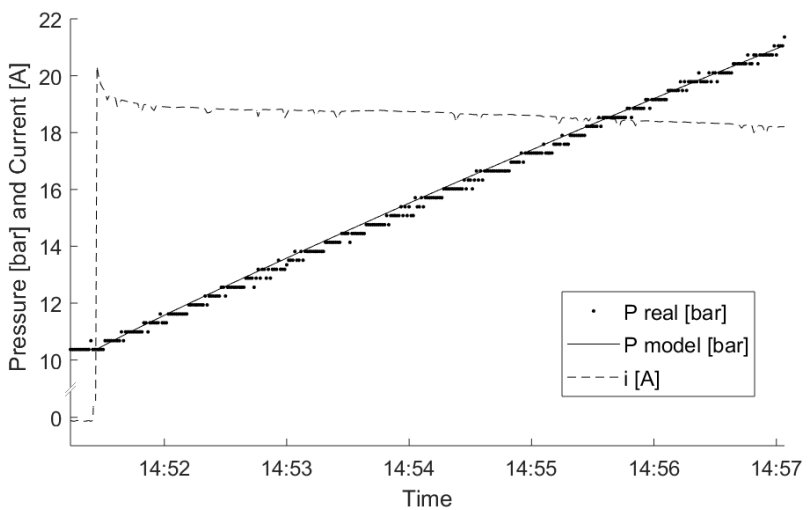

Fig. 4. Comparison of pressurization between the real system (dotted line) and the model (solid line)

increases $(t=600 \mathrm{~s})$. This fact is given due to the increment in the $H_{2}$ production. On the contrary, in response to a greater diffusivity $(t=1200 \mathrm{~s})$, the slope of the curve decreases slightly. This fact is given since more gas flows from the cathode chamber to the anodic chamber and, therefore, it does not go to the corresponding chamber. On the other hand, the level of solution increases with the growing presence of bubbles from the gases that were produced. Since the bubbles begin to detach from the free surface, the volume stops increasing and, on the contrary, begins to decrease due to the slow consumption of water in the electrochemical process. It should be noted that these changes in level are still small and they take a short period of time, after a few hours of operation.

As a validation start, a pressurization period of the real system was taken between 10 and 20 bar with a constant current. In Figure 4, the measured current input and the comparison between the recorded and the model pressures is illustrated. It can be observed that these values are in suitable agreement.

\section{CONCLUSIONS}

A phenomenological based semi-physical model of hydrogen production in an alkaline self-pressurized electrolyzer was proposed. Inherent characteristics of this kind of model provides additional information concerning phenomena taking place in the process. This fact allows further analysis to be made, e.g., controllability, observability and identifiability. Such information can be used to have a better understanding of the electrolyzer design and operation, with the added capability of a possible model-based controller synthesis for this equipment. The proposed model is capable of predicting level, pressure and all the concentrations in the system. The next step is to identify the model parameters in accordance with actual data taken from the real eletrolyzer.

\section{REFERENCES}

[1] T. Mahlia, T. Saktisahdan, A. Jannifar, M. Hasan, and H. Matseelar, "A review of available methods and development on energy storage: technology update," Renewable and Sustainable Energy Reviews, vol. 33, pp. 532-545, 2014.
[2] Electricity Storage FactBook, White paper, SBC Energy Institute, 2013, http://energystorage.org/resources/sbc-energy-instituteelectricity-storage-factbook.

[3] K. Onda, T. Kyakuno, K. Hattori, and K. Ito, "Prediction of production power for high-pressure hydrogen by high-pressure water electrolysis," Journal of Power Sources, vol. 132, pp. 64-70, 2004.

[4] O. Ulleberg, "Modeling of advanced alkaline electrolyzers: a system simulation approach," International Journal of Hydrogen Energy, vol. 28, pp. 21-33, 2003.

[5] H. Barthels, W. Brocke, K. Bonhoff, H. Groehn, G. Heuts, M. Lennartz, H. Mai, J. Mergel, L. Schmid, and P. Ritzenhoff, "PhoebusJülich: an autonomous energy supply system comprising photovoltaics, electrolytic hydrogen, fuel cell," International Journal of Hydrogen Energy, vol. 23, pp. 295-301, 1998.

[6] E. Amores, J. Rodríguez, and C. Carreras, "Influence of operation parameters in the modeling of alkaline water electrolyzers for hydrogen production,' International Journal of Hydrogen Energy, vol. 39, pp. $13063-13078,2014$.

[7] A. Ursúa, "Hydrogen production from water electrolysis: Current status and future trends," Proceedings of the IEEE, vol. 100, pp. 410426, 2012.

[8] K. Havre, P. Borg, and K. Tøysteinmmerberg, "Modelling and control of pressurized electrolyzer for operation in stand alone photovoltaic hydrogen power systems," in 2nd nordic symposium on hydrogen and fuel cells for energy storage, January 19-20 1995.

[9] A. Roy, S. Watson, and D. Infield, "Comparison of electrical energy efficiency of atmospheric and high-pressure electrolysers," International Journal of Hydrogen Energy, vol. 31, pp. 1964-1979, 2006.

[10] W. Hug, J. Divisek, J. Mergel, W. Seeger, and H. Steeb, "Intermittent operation and operation modelling of an alkaline electrolyzer," International Journal of Hydrogen Energy, vol. 18, no. 12, pp. 973-977, 1993.

[11] M. Schalenbach, A. R. Zeradjanin, O. Kasian, S. Cherevko, and K. J. Mayrhofer, "A perspective on low-temperature water electrolysis challenges in alkaline and acidic technology," International Journal of Electrochemical Science, vol. 13, pp. 1173-1226, 2018.

[12] M. Schalenbach, W. Lueke, and D. Stolten, "Hydrogen diffusivity and electrolyte permeability of the zirfon perl separator for alkaline water electrolysis," Journal of the Electrochemical Society, vol. 14, pp. 1480-1488, 2016.

[13] M. Schalenbach, M. Carmo, J. Fritz, David L.Mergel, and D. Stolten, "Pressurized pem water electrolysis: Efficiency and gas crossover," International Journal of Hydrogen Energy, vol. 38, pp. 14 921- 14933 , 2013.

[14] E. Hoyos, D. López, and H. Alvarez, "A phenomenologically based material flow model for friction stir welding," Materials \& Design, vol. 111 , pp. 321 - 330, 2016.

[15] C. Zuluaga-Bedoya, M. Ruiz-Botero, M. Ospina-Alarcón, and J. Garcia-Tirado, "A dynamical model of an aeration plant for wastewater treatment using a phenomenological based semi-physica modeling methodology,' Computers \& Chemical Engineering, vol. 117 , pp. $420-432,2018$.

[16] H. Alvarez, R. Lamanna, P. Vega, and S. Revollar, "Metodología para la obtención de modelos semifísicos de base fenomenológica aplicada a una sulfitadora de jugo de caña de azúcar," Revista Iberoamericana de Automática e Informática Industrial, vol. 6, pp. 10-20, 2009.

[17] M. David, H. Álvarez, R. Sánchez-Peña, and C. OcampoMartínez, "Detailed model deduction for an alkaline selfpressurized electrolyzer," https://sites.google.com/a/unal.edu.co/grupokalman/detailed-models-deduction, 2018. 